\title{
Estudo da Associação entre o Índice de Massa Corpórea (IMC) e Osteoartrite (OA) de Mãos $^{(*)}$
}

\section{Association between Bone Mass Index (BMI) and Hand Osteoarthritis (OA)}

\author{
Fernanda Ximenes Coimbra $^{(1)}$, Adil Muhib Samara ${ }^{(2)}$ e Ibsen Bellini Coimbra ${ }^{(3)}$
}

\section{RESUMO}

Objetivo: avaliar a incidência de osteoartrite de mãos nos pacientes encaminhados aos ambulatórios de reumatologia do Hospital das Clínicas da Universidade Estadual de Campinas e analisar a possível associação entre osteoartrite (OA) de mãos e o índice de massa corpórea (IMC). Métodos: foram analisados 515 pacientes, calculandose o IMC e também a idade no momento do diagnóstico (20-80 anos), sexo, cor e o diagnóstico através de regressão logística. Resultados: OA de mãos esteve presente em 15,8\% dos diagnósticos reumatológicos, incluindo-se, entre outras, as doenças do tecido conjuntivo e as doenças auto-imunes. Não houve relação estatisticamente significativa entre IMC e OA de mãos na regressão univariada. Entretanto, quando realizado estudo de regressão logística multivariada, verificou-se que a cada $1 \mathrm{~kg} / \mathrm{m}^{2}$ de aumento do IMC, o risco de OA de mãos aumenta em 5,4\%. Observou-se, ainda, que pacientes com sobrepeso apresentam 2,16 vezes mais risco de ter OA de mãos que os com peso considerado normal. Os obesos apresentam esse risco 2,04 vezes maior. Conclusões: a despeito de a associação entre o IMC e a prevalência da OA de mãos não ter sido significativa, pacientes com IMC $>25$ apresentam maior risco de desenvolverem OA de mãos.

Palavras-chave: osteoartrite $(\mathrm{OA})$ de mãos, índice de massa corpórea (IMC), fatores de risco.

\section{INTRODUÇÃO}

A osteoartrite (OA) ou osteoartrose, como é conhecida em nosso meio, ou ainda doença articular degenerativa, caracteriza-se por alterações da cartilagem decorrentes da quebra do equilíbrio entre a produção e a destruição dos elementos que compõem a sua matriz extracelular. Envolve

\begin{abstract}
Objective: To evaluate the incidence of hand osteoarthritis (OA) among patients that were referred to the Rheumatology Unit at State University of Campinas and to verify the association between hand $O A$ and bone mass index (BMI). Methods: We evaluated 515 outpatients, calculating the BMI and registering the age at diagnosis, sex, ethnicity and diagnosis. Logistic regression was also performed. Results: Hands $O A$ was present in $15.8 \%$. There was no relation between $B M I$ and hands $O A$ in the univariated analysis. However, when multivariated logistic regression was performed, we observed $5.4 \%$ increase in the risk to develop hands $O A$ per each $1 \mathrm{~kg} / \mathrm{m}^{2}$ of enhance in the BMI. We also observed that overweight patients showed 2,16 fold more risk than normal subjects to present hands $O A$. The obese patients showed 2.04 fold more risk than the normal ones. Conclusions: We concluded that despite the lack of direct association between $B M I$ and the hands $O A$ prevalence, patients with $B M I>25 \mathrm{~kg} / \mathrm{m}^{2}$ are at higher risk to develop hands $O A$.
\end{abstract}

Keywords: Hands osteoarthritis, Body Mass index, Risk factors.

ainda a participação do osso subcondral e da membrana sinovial. Dentre as patologias articulares que acometem os idosos, a OA é a mais freqüente. Estima-se que um terço dos indivíduos adultos com idade entre 25 e 74 anos apresentem evidências radiográficas de OA em pelo menos uma

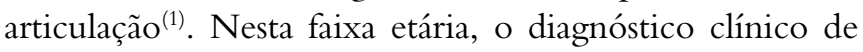
$\mathrm{OA}$, isto é, com quadro clínico estabelecido, ocorreu em

\footnotetext{
* Disciplina de Reumatologia da Faculdade de Ciências Médicas da Universidade Estadual de Campinas (FCM/Unicamp). Recebido em $31 / 07 / 2003$. Recebido em 20/04/2002. Aprovado, após revisão, em 28/10/2003.

1. Disciplina de Reumatologia da FCM/Unicamp.

2. Professor titular da disciplina de reumatologia do departamento de clínica médica da FCM/Unicamp.

3. Disciplina de Reumatologia da FCM/Unicamp.
}

Endereço para correspondência: Ibsen Bellini Coimbra. Av. Dr. Luiz de Tella 1033, Cid. Universitária, CEP 13083-000, Campinas, SP. 
$12 \%$ de cerca de 7.000 indivíduos avaliados (NHANES-I) ${ }^{(2)}$. No estudo Framingham Osteoarthritis Study Group, estimase que a prevalência da OA radiológica seja de um terço dos indivíduos com idade entre 63 e 93 anos e a OA sintomática ocorra em $9,5 \%$ do total dessa população( ${ }^{(3)}$.

Com relação à etiologia, há evidências que sugerem um envolvimento multifatorial, podendo ocorrer por influências genéticas, como os nódulos de Heberden na OA de mãos, distúrbios metabólicos, hormonais, idade avançada, raça e pela participação de citocinas inflamatórias. Pode ser também resultante da combinação destes vários fatores de risco.

Os hormônios sexuais são considerados como possíveis coadjuvantes no processo de surgimento de OA de mãos, particularmente em mulheres. Há dados que sugerem que o estrógeno, que atua mediado por citocinas e fatores de crescimento, desempenharia papel importante na OA de mãos ${ }^{(3)}$. Estas evidências incluem a maior prevalência desta entre as mulheres, além da maior incidência no período perimenopausa ${ }^{(3)}$. Também se evidencia a relação entre a maior prevalência de OA com possíveis marcadores de exposição exógena ao estrogênio, como na obesidade ${ }^{(3,4)}$.

A obesidade vem ganhando maior destaque entre os fatores metabólicos. $\mathrm{O}$ excesso de peso no desenvolvimento da OA de joelhos já é bem conhecido ${ }^{(5-8)}$. Também foi demonstrada uma associação positiva entre sobrepeso e surgimento da enfermidade na OA de quadril, embora esta associação não seja tão clara quanto à observada com relação aos joelhos ${ }^{(9)}$. Há estudo que sugere que o mecanismo através do qual o excesso de peso pode levar à OA esteja relacionado com o aumento da força sobre as articulações e com os fatores sistêmicos presentes na circulação das pessoas obesas $^{(10,11)}$. Possivelmente, estes mecanismos estariam ligados à presença de um fator de crescimento da cartilagem, um fator que pudesse acelerar a sua destruição ou, ainda, afetar o osso subcondral, levando à $\mathrm{OA}^{(10,11)}$. Em muitos casos, entretanto, a obesidade poderia ser subseqüente à $\mathrm{OA}$, onde se ganha peso por inatividade em conseqüência da dor. No caso da OA das mãos, a obesidade é provavelmente um fator que antecede a doença, já que suas articulações não suportam carga ${ }^{(12)}$.

Nas mãos, a maior parte da força sobre a articulação é resultante da contração muscular não relacionada com o peso; não há um aumento proporcional da força sobre a articulação com o aumento do peso. Assim sendo, tornase difícil explicar o resultado de um estudo que demonstrou associação direta entre obesidade e OA de mãos ${ }^{(12)}$. Nele, os autores avaliaram 25 homens obesos, clínica e radiolo- gicamente através de uma vista póstero-anterior das mãos, procurando-se OA. Nesta avaliação foram considerados quatro graus possíveis de gravidade. Todos os pacientes apresentavam obesidade mórbida por vários anos. Observou-se que as articulações interfalangianas distais apresentavam mais sinais de OA em homens corpulentos. De 40 articulações das mãos avaliadas, 14 apresentavam grau 0 (nenhum ), 25 grau 1 ( mínimo ), 1 grau 2 ( moderado) e nenhum dos homens apresentaram grau grave ou grau 4.

Num outro estudo ${ }^{(13)}$, investigou-se o papel da obesidade na etiologia da OA e descobriu-se que a incidência de mãos se eleva com o aumento no índice de massa corpórea. Desse estudo com 4.795 indivíduos com início em 1962, foram reexaminados 1.276 dos participantes. Destes, 588 homens e 688 mulheres com 50 a 74 anos de idade, avaliaram-se 22 anos depois radiografias de 32 articulações de mãos e punhos. Deles, 40,9\% novos casos de OA de mãos se desenvolveram durante aquele período. As mulheres tiveram uma incidência significativamente maior e eram 2,6 vezes mais obesas que os homens. Essas mulheres também apresentaram um risco de 1,9 vezes maior de desenvolver OA quando comparadas às normais. $\mathrm{O}$ estudo revelou ainda que o excesso de peso não estaria associado com uma maior gravidade da osteoartrite de mãos.

Em 1996, outros autores ${ }^{(14)}$ estudaram 329 pares de mulheres gêmeas, monozigóticas e dizigóticas, com idades entre 48 e 70 anos, sem diferenças significativas com relação à altura, uso de cigarro, idade da menopausa e uso de estrógenos entre elas. Os pares de gêmeas que apresentaram diferenças entre si com relação à presença de OA foram analisados separadamente e observou-se que a obesidade foi um importante fator de risco para OA de articulações fêmoro-tibial, patelo-femoral e metacarpofalangianas (MCF). Mostrou-se também que, para cada quilograma $(\mathrm{kg})$ a mais no peso de uma gêmea, o risco de desenvolver OA de MCF aumentava entre 9\% e 13\%, comparando-se com seu gêmeo. Gêmeas com a doença são geralmente 3 a 5 quilos mais pesadas que seus irmãos.

A idade é um fator que influencia a gravidade da OA, especialmente em mulheres ${ }^{(10)}$. Num estudo inglês, analisou-se mais de 2.000 indivíduos e observou-se o aumento significativo da gravidade de OA nas idades mais avançadas ${ }^{(15)}$.

Outros estudos populacionais mostraram também a importância do fator raça na etiologia da $\mathrm{OA}^{(16,17)}$. Demonstrou-se que a prevalência e distribuição de OA é variável dependendo do grupo étnico, com alta prevalência de OA em brancos quando comparados aos africanos, asiáticos e hispânicos. Entretanto, posteriormente em outra casuística, 
demonstraram-se relações comparáveis de OA de mãos entre americanos de origem africana e mulheres brancas ${ }^{(18)}$.

Este trabalho se destina a avaliar a incidência de OA de mãos entre os pacientes encaminhados aos ambulatórios de Reumatologia do Hospital das Clínicas da Universidade Estadual de Campinas, analisando-se a possível associação de OA de mãos e o índice de massa corpórea.

\section{PACIENTES E MÉTODOS}

Foram analisados 515 pacientes encaminhados ao ambulatório de triagem da Reumatologia do Hospital das Clínicas da Universidade Estadual de Campinas (conforme cálculo amostral realizado a partir de estudo piloto com 42 pacientes) ${ }^{(19)}$.

Todos foram pesados e medidos. Com estas duas variáveis foi calculado o índice de massa corpórea (IMC), que é a relação entre o peso e o quadrado da altura.

Os pacientes foram analisados quanto à idade no momento do diagnóstico (de 20 a 80 anos), quanto ao sexo e também quanto à cor, considerando-se brancos aqueles que apresentavam pais e avós brancos e não-brancos os demais.

Foram ainda analisados os diagnósticos clínicos numa consulta de triagem e acompanhados até sua confirmação. O diagnóstico de OA de mãos foi realizado clinica e radiologicamente.

\section{ANÁLISE DOS RESULTADOS}

Os dados obtidos nesta avaliação, conforme sugerido pelo cálculo de tamanho amostral realizado pelo serviço de estatística da comissão de pesquisa da Faculdade de Ciências Médicas (FCM), onde $\alpha=5 \%$ e $\beta=10 \%{ }^{(15)}$, foram analisados através de regressão logística, tendo sido definido no cálculo amostral que:

- para estimar um odds-ratio de 3.0 quando o IMC for igual à média acrescida de um desvio padrão (valores do piloto), seriam necessários 162 casos;

- para estimar um odds-ratio de 2.0 quando o IMC for igual à média acrescida de um desvio padrão (valores do piloto), seriam necessários 315 casos.

- $\mathrm{Na}$ análise das outras variáveis, estabeleceu-se a freqüência em que ocorriam na $\mathrm{OA}$ de mãos.

\section{RESULTADOS}

Dentre os pacientes $(n=515)$ que procuraram o serviço de triagem de Reumatologia do Hospital da Unicamp entre o período de 04/01/1999 e 17/02/2000, observou-se que OA de mãos esteve presente em 15,8\% entre todos os diagnósticos reumatológicos, incluindo-se as doenças do tecido conjuntivo, doenças auto-imunes, etc (Figura 1). Entre todos os diagnósticos de OA realizados, as mãos atingiram a significativa porcentagem de $51 \%$.
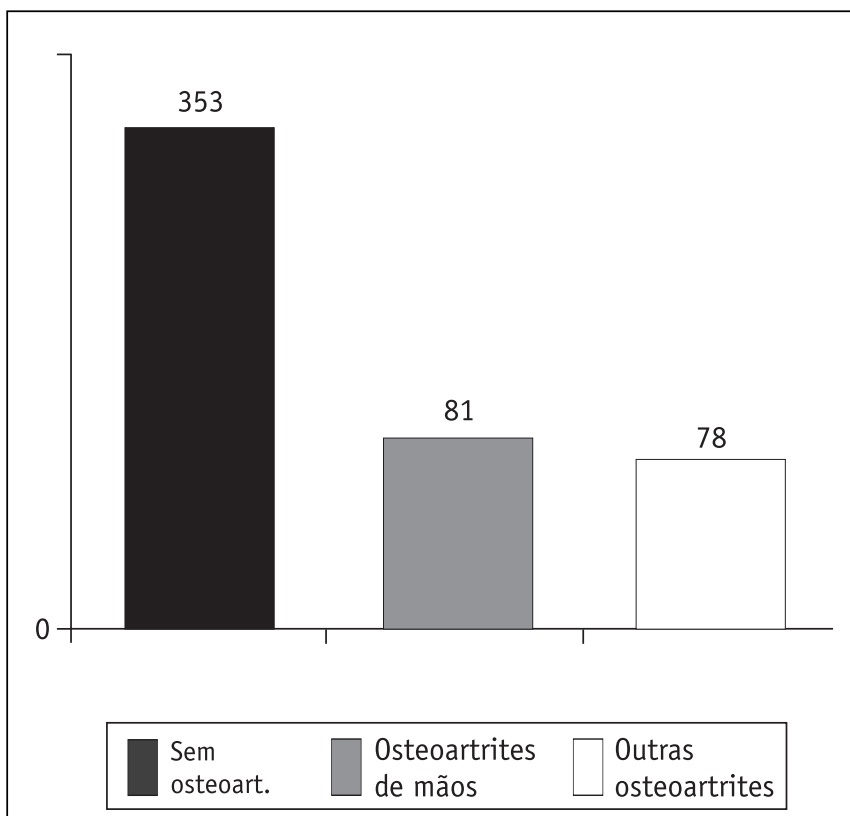

Obs.: Não foram consideradas informações sobre três pacientes

FiguRa 1 - Freqüência dos diagnósticos.

\section{RELAÇÃO ENTRE IMC E OA DE MÃOS}

Não houve relação estatisticamente significativa entre IMC e OA de mãos. Após estudo de regressão logística univariada para a presença ou não de $\mathrm{OA}$ de mãos com relação às variáveis pesquisadas, não se observou relação entre o peso e OA de mãos, o mesmo ocorrendo entre IMC e OA de mãos (Tabela 1). Quando realizado estudo de regressão logística multivariada (utilizando-se o critério "Stepwise" para seleção de variáveis), verificou-se que a cada $1 \mathrm{~kg} / \mathrm{m}^{2}$ de incremento do IMC, o risco de OA de mãos aumenta em 5,4\%, ou seja, há 1,054 vez mais chances de OA de mãos que ausência (Tabela 2).

Com o intuito de analisar a chance da presença de OA de mãos com relação às três principais categorias de IMC (IMC $<25$ indivíduo normal; IMC entre 25 e 30, indivíduo com sobrepeso e IMC entre 30 e 40, obeso), a regressão logística univariada para a presença ou não de OA de mãos foi refeita. Foram então criadas novas variáveis IMCA e IMCB (respectivamente presença e ausência de OA de 
mãos). Foram então classificados em normais (IMCA $=0$, IMCB $=0)$, sobrepeso $(\mathrm{IMCA}=0, \mathrm{IMCB}=1)$ e obesos $(\mathrm{IMCA}=1, \mathrm{IMCB}=0)$. A partir desta análise, observou-se que pacientes com sobrepeso apresentam 2,16 vezes mais risco de ter OA de mãos que os normais e que os obesos apresentam 2,04 vezes mais risco de ter OA de mãos que os normais (Tabela 3 ).

\section{ANÁlise dA PRESENÇA OU AUSÊNCIA DA OA DE MÃOS COM RELAÇÃO ÀS OUTRAS VARIÁVEIS}

Sexo: A análise dos dados mostrou que nesta série de pacientes não existe uma freqüência maior de $\mathrm{OA}$ de mãos no sexo feminino. Os dados podem ser observados na Figura 2.

Idade: $\mathrm{Na}$ análise da variável idade determinou-se que, numa ordem escalonada, a maior incidência foi observada na quinta, sexta e sétima décadas. Na sétima década considerou-se a incidência com relação à população idosa existente (Figura 3).

Etnia: $87,65 \%$ dos casos ocorreram entre pacientes de raça branca (Figura 4 ).

TABELA 1

RESUlTADOS DA ANÁLISE DE REGRESSÃO LOGÍSTICA UNIVARIADA PARA OA DE MÃOS

\begin{tabular}{lccc}
\hline Variáveis & $\boldsymbol{P}$ & Odds Ratio & I.C. 95\% 0dds Ratio \\
\hline Raça & 0,2318 & 1,548 & $0,787-3,341$ \\
Altura & 0,8284 & 1,487 & $0,040-53,419$ \\
Peso & 0,0576 & 1,018 & $0,999-1,037$ \\
IMC & 0,0602 & 1.051 & $0,998-1,108$ \\
\hline
\end{tabular}

Odds Ratio: razão de risco; IC: intervalo de confiança

Obs.: não foram consideradas os HD Outras-OA, apenas HD 'Não-OA' vs 'OA Mãos'.

TABELA 2

RESUltadOS DA ANÁLISE DE REGRESSÃO LOGÍSTICA MULTIVARIADA PARA OA DE MÃOS

\begin{tabular}{lccc}
\hline Variáveis & $\boldsymbol{P}$ & 0dds Ratio & I.C. 95\% 0dds Ratio \\
\hline Intercepto & 0,0003 & --- & --- \\
IMC & 0,0501 & 1,054 & $1,000-1,111$ \\
\hline
\end{tabular}

Obs.: não foram consideradas os HD's Outras-OA, apenas HD 'Não-OA' vs 'OA Mãos'.

TABELA 3

RESUltAdOS DA ANÁLISE DE REGRESSÃO LOGÍ́STICA UNIVARIADA PARA OA DE MÃOS

\begin{tabular}{lccc}
\hline Variáveis & $\boldsymbol{P}$ & Odds Ratio & I.C. 95\% 0dds Ratio \\
\hline Intercepto & 0,0001 & --- & --- \\
IMCA & 0,0559 & 2,046 & $0,988-4,328$ \\
IMCB & 0,0292 & 2,163 & $1,095-4,416$ \\
\hline
\end{tabular}

Rev Bras Reumatol, v. 44, n. 3, p. 206-11, mai./jun., 2004

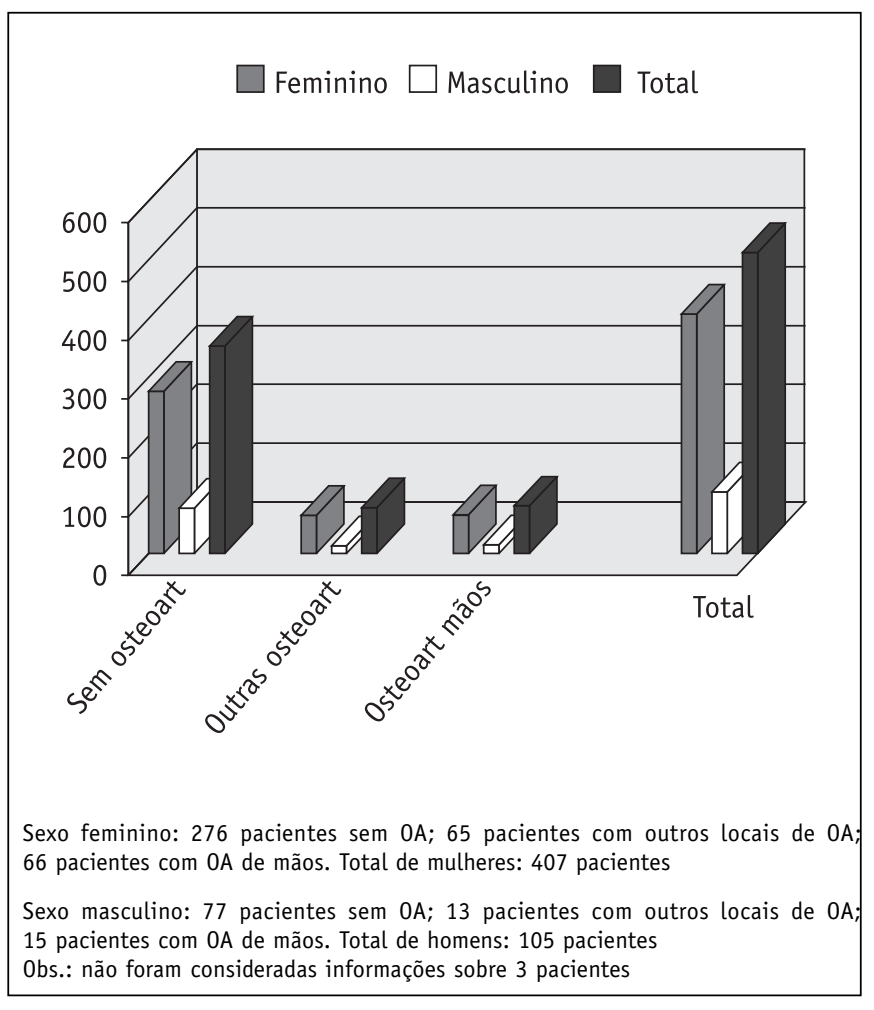

Figura 2 - Freqüência para sexo versus diagnósticos.

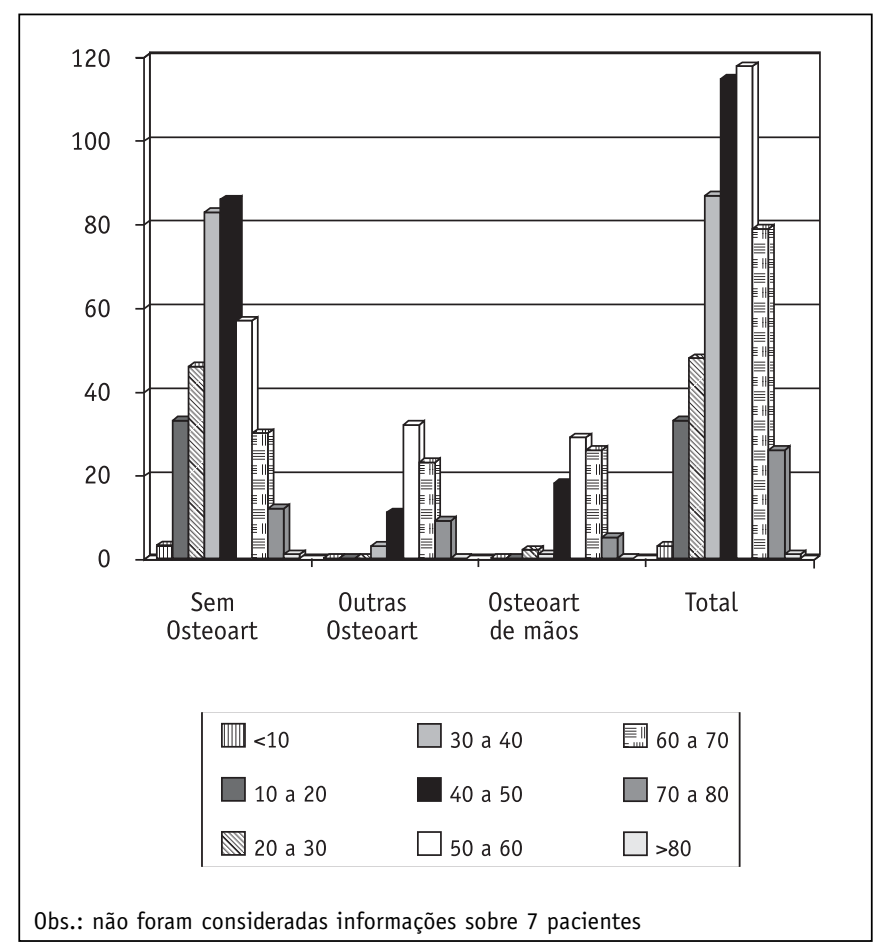

FIgURA 3 - Freqüência para idade versus diagnósticos. 


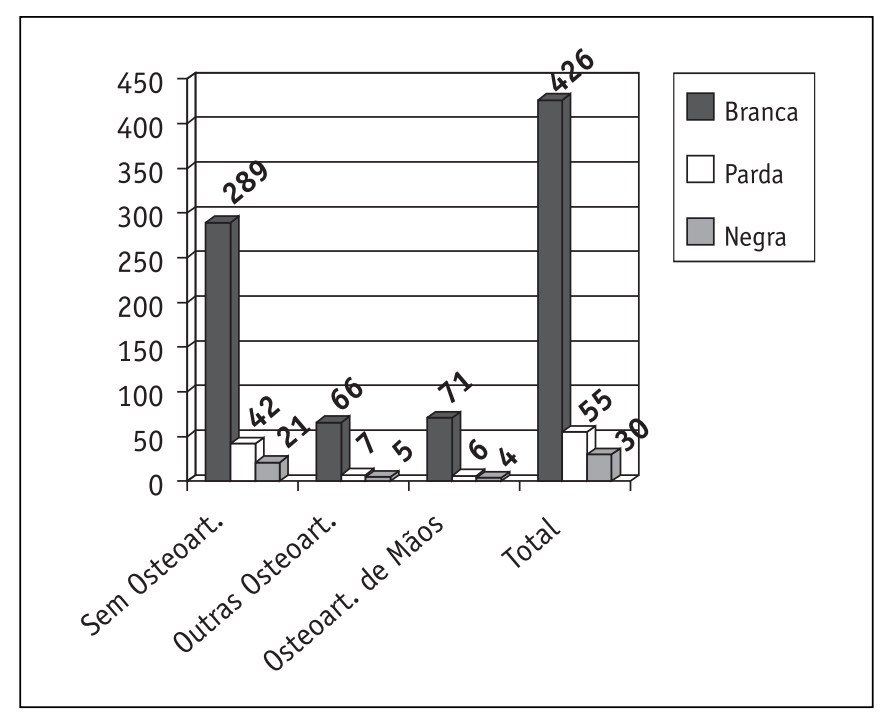

FIgURA 4 - Freqüência de raça versus hipótese diagnóstica.

\section{DISCUSSÃO}

A participação do excesso de peso no desenvolvimento da OA de joelhos já está bem estabelecida, além de ter sido demonstrada também associação positiva entre a presença de sobrepeso e o surgimento da OA de quadril, a despeito de esta não ser tão clara quanto à observada com relação aos joelhos. Também tem sido observada associação da presença de obesidade e OA das mãos. Homens com obesidade mórbida mostram a sua associação direta com a osteoartrite das mãos ${ }^{(12)}$. Um outro estudo, relativo ao papel da obesidade na etiologia da OA mostrou que sua incidência nas mãos se eleva com o aumento no índice de massa corpórea ${ }^{(13)}$. $\mathrm{Na}$ Inglaterra, mulheres gêmeas, monozigóticas e dizigóticas, com idades entre 48 e 70 anos, apresentaram diferenças entre si com relação à presença de $\mathrm{OA}$, porém pareadas com relação à altura, uso de cigarro, idade da menopausa e uso de estrógenos, observando-se que a obesidade foi um forte fator de risco para OA de articulações tíbio-femoral, patelofemoral, e metacarpofalangianas $(\mathrm{MCF})^{(14)}$. Esta associação, no entanto, até o momento não havia sido demonstrada em nenhuma série de pacientes brasileiros.

Neste trabalho foram realizadas regressões logísticas uni e multivariadas, analisando-se a associação entre IMC e a prevalência da OA de mãos. Apenas a regressão multivariada mostrou uma significativa relação entre elas. Esses resultados são discrepantes com aqueles encontrados em um trabalho de1955(20), que não demonstrou associação entre nódulos de Heberden e obesidade e também com os de um outro, em $1959^{(21)}$, que relatou uma pequena evidência desta associação. Deve-se considerar também que a amostra aqui estudada era substancialmente maior. Em nossos achados, concluiu-se que aqueles considerados sobrepesos (IMC entre 25-30) têm 2,16 vezes mais risco de apresentar OA de mãos que os de peso normal $(\mathrm{IMC}<25)$. Um estudo de $1996^{(11)}$ também encontrou resultados semelhantes e concluiu-se que o IMC $<25$ geralmente reduz o risco de incidência da doença, presumindo servir como conduta terapêutica.

Foram avaliadas também outras variáveis. Com respeito ao sexo, observou-se que a imensa maioria dos casos que procuraram o serviço no período do estudo era do sexo feminino $(79,49 \%)$ e da raça branca $(87,65 \%)$, em concordância com outros estudos ${ }^{(12,22,23)}$, que também chegaram à conclusão que no sexo feminino existe uma freqüência maior de OA de mãos e especularam sobre a possível relação entre os estrógenos e a enfermidade, considerando-se ser a cartilagem um tecido estrógeno dependente ${ }^{(24)}$. Estes autores postulam que os estrógenos poderiam afetar diretamente esse tecido porque há receptores deles nos condrócitos ou porque atuariam via sinalizadores secundários ${ }^{(24)}$. Além disso, já se demonstrou que o estrógeno pode atuar como modificador dos níveis de citocinas, especialmente a IL-6, afetando o metabolismo da cartilagem articular ${ }^{(25)}$. A prevalência de OA de mãos em idades avançadas corrobora com esta hipótese. Especula-se ainda a possibilidade de o tecido adiposo desempenhar função ainda não elucidada no metabolismo da cartilagem, especialmente em mulheres menopausadas, o que poderia explicar o maior risco para a incidência da doença ${ }^{(26)}$. Outros estudos mostraram que mulheres histerectomizadas apresentaram maior incidência e sinais clínicos mais graves que aquelas usadas como controle ${ }^{(27,28)}$.

Também neste estudo verificou-se uma maior incidência de OA de mãos a partir da sexta década de vida, em concordância com vários estudos da literatura, que indicam a idade como altamente relacionada com o seu desenvolvimento ${ }^{(22,29,30)}$.

Finalmente, conclui-se que OA de mãos foi o diagnóstico observado em 15,8\% dos pacientes encaminhados à Reumatologia neste serviço. A despeito da associação com o IMC e da prevalência da OA de mãos não ter sido significativa, confirmou-se que pacientes com IMC $>25$, apresentam maior risco de desenvolverem OA de mãos. 


\section{REFERÊNCIAS}

1. Lawrence RC, Hochberg MC, Kelsey JL, et al: Estimates of the prevalence of selected arthritic and musculoskeletal diseases in the United States. J Rheumatol 16: 427-41, 1989.

2. Maurek K: Basic data on arthritis knee, hip and sacroiliac joints in adults ages 25-74, United States,1971-1975. Vital Health Stat 213: S11, 1979.

3. Felson DT, Naimark A, Anderson J, Kazis L, Castelli W, Meenam RF: The prevalence of knee osteoarthritis in the elderly. Arthritis Rheum 30: 914-8, 1987.

4. Lethbridge-Cejku M, Tobin JD, Scott WW Jr, Reichle R, Plato $\mathrm{CC}$, Hochberg MC: The relationship of age and gender to prevalence and pattern of radiographic changes of osteoarthritis of the knee: data from Baltimore Longitudinal Study of Aging. Aging Clin Exp Res 6: 353-7, 1994.

5. Anderson JJ, Felson DT: Factors associated with osteoarthritis of the knee in the First National Health and Nutrition Examination Survey (NHANES-I): evidence for an association with overweight, race and physical demands of work. Am J Epidemiol 128: 179-80, 1988.

6. Davis MA, Ettinger WH, Neuhaus JM, Cho SA, Houck WW: The association of knee injury and obesity with unilateral and bilateral osteoarthritis of the Knee. Am J Epidemiol 130: 278-88, 1989.

7. Davis MA, Neuhaus JM, Ettinger WH, Mueller WH: Body fat distribution and osteoarthritis. Am J Epidemiol 132: 701-7, 1990.

8. Hochberg MC, Lethbridge-Cejku M, Scott WW Jr, Reichle R, Plato CC, Tobin JD: The association of body weight, body fatness and body fat distribution with osteoarthritis of the knee: data from the Baltimore Longitudinal Study of Aging. J Rheumatol 22: 488-93, 1995.

9. Tepper S, Hochberg MC: Factors associated with hip osteoarthritis: data from the First National Health and Nutrition Examination Survey (NHANES-I). Am J Epidemiol 137: 1081-8, 1993.

10. Caspi D, Flusser G, Farber I, et al: Clinical, Radiologic, Demographic, and Occupational Aspects of Hand Osteoarthritis in the Elderly. Semin Arthritis Rheum 30: 321-31, 2001.

11. Felson D: Weight and osteoarthritis. Am J Clin Nutr 63: 430S-2S, 1996.

12. Carman W, Sowers M, Hawthorne V, Weissfeld L: Obesity as a risk factor for osteoarthritis of the hand and wrist: A prospective study. Am J Epidemiol 139: 119-29, 1994.

13. Goldin R, McAdam L, Louie J, et al: Clinical and radiological survey of the incidence of osteoarthrosis among obese patients. Ann Rheum Dis 35: 349-53, 1976.

14. Cicuttini F, Baker J, Spector T: The association of obesity with osteoarthritis of the hand and knee in women: a twin study. J Rheumatol 23: 1221-6, 1996.
15. Lawrence JS, Bremner JM; Bier F: Osteoarthrosis. Prevalence in the population and relationship between symptoms and $\mathrm{x}$-rays changes. Ann Rheum Dis 25: 1-24, 1996.

16. Anderson JJ, Felson DT: Factors associated with osteoarthritis of the knee in the first National Health and Nutrition Examination Survey ( NHANES I ). Evidence for an association with overweight, race and physical demands of work. Am J Epidemiol 128: 179-89, 1988.

17. Solomon L: International Symposium on joint failure. Geographical and anatomical patterns of OA. Br J Rheumatol 23: 177-80, 1984.

18. Sowers M, Lachance L, Hochberg M, et al: Radiographically defined osteoarthritis of the hand and knee in young and middle aged African American and Caucasian women. Osteoathritis Cartilage 8: 69-77, 2000.

19. Hsieh FY: Sample Size Tables for Logistic Regression. Statistics in Medicine 8: 795-802, 1989.

20. Stecher RM: Heberden oration. Heberden`s nodes. A clinical description of osteoarthritis of the finger joints. Ann Rheum Dis 14 1-10, 1955.

21. Silberg M, Frank EL, Jarrer SR, et al: Aging and osteoarthritis of the human sternoclavicular joint. Osteoarthritis 35: 851-63, 1959.

22. Sahyoun NR, Hochberg MC, Helmick CG, Harris T, Pamuk ER: Incidence of Self-Reported Physician Diagnosed Arthritis Among Women. Am J Public Health 89: 391-4, 1999.

23. Sowers D, Zobel D, Weissfeld L, et al: Progression of osteoarthritis of the hand and metacarpal bone loss. Arthritis Rheum 34: 36-42, 1991.

24. Tsai CL, Liu TK: Osteoarthritis in women: its relationship to estrogen and current trends. Life Sci 50: 1737-44, 1992.

25. Guerne PA, Carson D, Lotz M: IL-6 production by human articular chondrocytes. Modulation of its synthesis, growth factors, and hormones in vitro. J Immunol 144: 499-505, 1990.

26. Pacifici R, Avioli LV: The effect of natural and surgical menopause on the secretion of cytokenis from human blood monocytes. Osteoporosis Int: S106-7, 1993.

27. Spector TD, Hart DJ, Brown P: Frequency of osteoarthritis in hysterectomized women. J Rheumatol 18: 1877-83, 1991.

28. Samantha A, Jones A, Regan M, Wilson S, Doherty: In osteoarthritis in women affected by hormonal changes or smoking? $\mathrm{Br} \mathrm{J}$ Rheumatol 32: 366-70, 1993

29. Van Saase JLCM, Van Romunde LKJ, Cats A, Vandenbroucke JP, Valkenburg HA: Epidemiology of osteoarthritis: Zoetermeer survey. Ann Rheum Dis 48: 271-80, 1989.

30. Yazici H, Saville PD, Salvati EA, et al: Primary osteoarthrosis of the knee or hip: Prevalence of Heberden`s nodes in relation to age and sex. JAMA 231: 1256-60, 1975. 